Reprod. Nutr. Dévelop., 1988, 28 Suppl. n¹, 59-60

\title{
Influence des quantités de matière sèche offerte sur l'ingestion des graminées tropicales
}

D. RICHARD, H. GUERIN (*), G. ROBERGE, D. FRIOT

Laboratoire national de l'Elevage et de Recherche vétérinaire B.P. 2057, Dakar, Sénégal.

$\left.{ }^{*}{ }^{*}\right)$ Institut d'Elevage et de Médecine vétérinaire des Pays tropicaux, 10, rue Pierre-Curie, 94704 Maisons-Alfort Cedex, France.

Summary. The dry matter intake (DMI) by sheep of 69 samples of tropical grasses was positively influenced by the amount of dry matter offered (DMO) and the proportion of refusal allowed. The introduction of DMO as independant variable improved the prediction of the DMI from forage age and lignin content.

Les mesures de digestibilité des fourrages verts peuvent être faites en alimentant les moutons ad libitum, de façon à mesurer en même temps l'ingestibilité des fourrages. En zone tempérée, les taux de refus (rapport de la quantité de matière sèche non consommée sur la quantité de matière sèche offerte) sont compris entre 5 et $15 \%$. En zone tropicale, il n'existe guère de règle ; les taux de refus varient de 5 à $7 \%$ (Minson, 1981) à $45 \%$ (Combellas et Gonzalez, 1973). Suite à une série d'essais de digestibilité sur des graminées tropicales, l'influence des quantités de matière sèche offerte (MSO) sur les quantités de matière sèche volontairement ingérée (MSVI) a été analysée.

Matériel et méthodes. Les mesures ont été effectuées sur des moutons à la station de Sangalkam dans la région du Cap vert au Sénégal et au C.R.Z. de Bouaké en Côte d'lvoire. Les résultats ci-dessous ne comprennent pas les mesures faites en saison froide au Sénégal, mais uniquement les essais de saison chaude au cours de laquelle les conditions climatiques sont voisines dans les deux stations. Les MSVI de repousses de 3 à 11 semaines de 4 graminées fertilisées et irriguées (Brachiaria mutica, Panicum maximum var. K 187 B et var. Sotuba, Pennisetum purpureum var. Kizozi) ont été mesurées lors d'essais de digestibilité dont la méthodologie a été précédemment décrite (Richard et al., 1987). Les taux de refus, choisis par les expérimentateurs qui se sont succédé, ont été variables pour un même âge de repousse et sont constatés a posteriori. Pour un même essai, les quantités distribuées en vert étaient constantes et les taux de refus, fixés dans certaines limites, étaient calculés à la fin des mesures.

Les fourrages offerts et refusés ont été analysés pour l'azote, la cellulose brute de Weende et les constituants pariétaux de Van Soest.

Résultats et discussion. Les principales caractéristiques des 69 fourrages distribués sont données dans le tableau 1.

Les MSVI sont significativement liées à l'âge du fourrage, à la digestibilité de la matière organique et aux teneurs en principaux constituants chimiques. Les 
TABL. 1. - Composition chimique des fourrages offerts et ingérés (en $\mathrm{g} / \mathrm{kg} \mathrm{MS}$ ).

\begin{tabular}{|c|c|c|c|c|c|}
\hline \multirow{7}{*}{$\begin{array}{l}\text { Matière sèche }(\mathrm{g} / \mathrm{kg} \text { vert }) \\
\text { Matières azotées totales } \\
\text { Cellulose brute } \\
\text { Parois cellulaires (NDF) } \\
\text { Lignocellulose (ADF) } \\
\text { Lignine }\end{array}$} & \multicolumn{3}{|c|}{$\begin{array}{c}\text { Fourrage offert }(\mathrm{O}) \\
\text { (moyenne, écart-type, } \\
\text { extrêmes) }\end{array}$} & \multicolumn{2}{|c|}{$\begin{array}{l}\text { Différence entre } \\
\text { le fourrage ingéré }(\mathrm{I}) \text { et } \\
\text { le fourrage offert (I-O) } \\
\text { (moyenne, écart-type) }\end{array}$} \\
\hline & 177 & (41) & $96-293$ & & \\
\hline & 106 & (32) & $61-201$ & +7 & (6) \\
\hline & 358 & (30) & $292-437$ & -10 & (9) \\
\hline & 718 & (40) & $613-802$ & -6 & $(16)$ \\
\hline & 425 & (38) & $325-485$ & -7 & (21) \\
\hline & 57 & (15) & $33-100$ & -4 & (5) \\
\hline
\end{tabular}

écart-types résiduels (ETR) des régressions entre la $M S V I$ et les différentes variables sont compris entre 6,4 et 9,2 g. L'ETR le plus faible est obtenu avec les teneurs en lignocellulose. La précision des relations est améliorée si les MSO ou les taux de refus sont pris comme seconde ou troisième variable explicative. Les MSO améliorent beaucoup plus la précision, de 2,6 à 3,9 g, que les taux de refus, de 0,1 à $1 \mathrm{~g}$. Ceci est également souligné par Zemmelink (1980). L'équation la plus précise est obtenue avec l'âge des repousses ( $\mathrm{J}$ en jours), la teneur en lignine ( $L$ en $\mathrm{g} / \mathrm{kg} M S$ ) et la $M S O: R^{2}=0,71$, ETR $=5,3$. La liaison est encore plus étroite si les 13 données relatives au Pennisetum sont retirées car sa faible teneur en matière sèche influe négativement sur la MSVI (Richard et al., 1987). L'équation est alors :

$$
\begin{aligned}
& \text { MSVI }=-0,24 \quad J-0,29 L+0,34 M S O+59,5 \quad R^{2}+0,79 \quad \text { ETR }=4,5, \\
& n=56
\end{aligned}
$$

Les quantités ingérées des graminées tropicales augmentent donc avec les MSO, ce qui est en accord avec les observations d'autres auteurs (Butterworth, 1965 ; Zemmelink, 1980). Un taux de refus élevé permet à l'animal de sélectionner les feuilles sur des fourrages qui en contiennent relativement peu. Cette sélection entraîne une teneur plus élevée en MAT et plus faible en constituants pariétaux du fourrage ingéré que celles du fourrage offert comme le montre le tableau 1.

L'influence des taux de refus paraît plus importante pour les graminées tropicales que pour les graminées tempérées. Ces taux doivent être supérieurs à $15 \%$ pour obtenir l'ingestion maximale des fourrages tropicaux. IIs doivent donc être précisés dans les résultats relatifs aux ingestions des fourrages tropicaux et méritent d'être standardisés : un taux compris entre 20 et $25 \%$ paraît recommandable.

Butterworth M. H., 1965. J. agric. Sci., 65, 233-239.

Combellas J., Gonzalez E., 1973. Agron. trop., 21, 277-286.

Minson D. J., 1981. In Wheeler J. L. and Mochrie R. D., Forage evaluation, concepts and techniques, CSIRO, Melbourne, 159-174.

Richard D., Friot D., Guerin H., Roberge G., 1987. Reprod. Nutr. Dévelop., 27, 195-196.

Zemmelink G., 1980. Centre for agricultural Publishing and Documentation, Wageningen, $100 \mathrm{p}$. 